\title{
SNIa, white dwarfs and the variation of the gravitational constant
}

\author{
J. Isern ${ }^{1,2}$, E. Garcia-Berro ${ }^{1,3}$ and P. Lorén-Aguilar ${ }^{1,3}$ \\ ${ }^{1}$ Institut d'Estudis Espacials de Catalunya (IEEC), Edifici Nexus, c/ Gran Capità 2, E-08034 \\ Barcelona, Spain \\ ${ }^{2}$ Institut de Ciències de l'Espai (CSIC), Torre C-5 Parell, Facultat de Ciències, Campus UAB, \\ E-08193 Bellaterra, Spain \\ email: isern@ieec.cat \\ ${ }^{3}$ Departament de Fisica Aplicada, Universitat Politècnica de Catalunya, c/Esteve Terrades, 5, \\ E-08860 Castelldefels, Spain \\ email: garcia@fa.upc.edu; email: loren@fa.upc.edu
}

\begin{abstract}
The critical role that the gravitational constant, $G$, plays in the Theory of General Relativity and the possibility, introduced by theories that unify gravity with other interactions, that $G$ could vary in time and space have bursted the interest in detecting such variations or, at least, to bound them as tightly as possible.

White dwarfs represent the last evolutionary stage of stars with masses smaller than $10 \pm$ $2 M_{\odot}$. Since their mechanical structure is sustained by the pressure of degenerate electrons, they do not radiate nuclear energy and their evolution is just a simple gravothermal cooling process. On the other hand, white dwarfs in close binary systems can accrete matter from the companion, experiencing nova outbursts, or in some cases they can also reach Chandrasekhar's mass and explode as a Type Ia supernova (SNIa). Since the cooling of single white dwarfs and the properties of SNIa strongly depend on the precise value of $G$ and on its possible secular variation, white dwarfs can be used to constrain such hypothetical variations.

When white dwarfs are cool enough, their luminosity is entirely of gravothermal origin. Any variation of $G$ modifies the energy balance of their interiors and, consequently, also modifies their luminosity. Formally, the influence of a secular variation of $G$ can be expressed as $L=$ $-\dot{B}+\Omega(\dot{G} / G)$ where $B=U+\Omega$ is the total binding energy, $U$ is the total internal energy and $\Omega$ is the gravitational energy. Thus, if $\dot{G} \neq 0$ the luminosity is modified and the characteristic cooling time is different from that obtained in the case in which $\dot{G}=0$. Detecting such variations can be done using the luminosity function of white dwarfs, which is defined as the number of white dwarfs of a given luminosity per unit of magnitude interval. It increases monotonically with the magnitude and displays a sharp cut-off due to the finite age of the Galaxy. The position of this cut-off is sensitive to the age of the Galaxy and to the value of $\dot{G}$ and, thus, it can be used to obtain a bound. White dwarfs also display $g$-mode pulsations driven by the $\kappa$-mechanism and the period of pulsation experiences a secular drift of $\dot{P} / P \simeq-a \dot{T} / T+b \dot{R} / R$, where $a$ and $b$ are model-dependent constants of the order of unity. Since both $\dot{T}$ and $\dot{R}$ depend on $\dot{G}$, the measure of $\dot{P}$ can also provide useful constraints.

Type Ia supernovae are thought to be the result of the thermonuclear explosion of a carbonoxygen white dwarf with a mass near Chandrasekhar's limit in a close binary system. The peak luminosity of SNIa is proportional to the mass of nickel synthesized which can be considered as a fixed fraction of the mass of the exploding star, $M_{\mathrm{Ni}} \propto M_{\mathrm{Ch}}=(\hbar c)^{3 / 2} / m_{\mathrm{p}} G^{3 / 2}$. Therefore the properties of this peak with redshift can be used to test the variation of $G$ with cosmic ages.

Although the bounds obtained in these ways have been currently superseeded by other more accurate methods, when the ongoing surveys searching for SNIa and white dwarfs will be completed, the expected bounds will be as tight as $\sim 10^{-13} \mathrm{yr}^{-1}$.
\end{abstract}

Keywords. Gravitation, Stars: white dwarfs, supernovae 